\title{
ELEMENTARY APPROACH TO CLOSED BILLIARD TRAJECTORIES IN ASYMMETRIC NORMED SPACES
}

\author{
ARSENIY AKOPYAN, ALEXEY BALITSKIY, ROMAN KARASEV, \\ AND ANASTASIA SHARIPOVA \\ (Communicated by Patricia Hersh)
}

\begin{abstract}
We apply the technique of Károly Bezdek and Daniel Bezdek to study billiard trajectories in convex bodies, when the length is measured with a (possibly asymmetric) norm. We prove a lower bound for the length of the shortest closed billiard trajectory, related to the non-symmetric Mahler problem. With this technique we are able to give short and elementary proofs to some known results.
\end{abstract}

\section{INTRODUCTION}

In this paper we consider billiards in convex bodies and estimate the minimal length of a closed billiard trajectory. This kind of estimate is rather useful in various practical applications; see further references on this subject in [5].

In 4. Shiri Artstein-Avidan and Yaron Ostrover presented a unified symplectic approach to handle billiards in a convex body $K \subset V$ (here $V$ is a real vector space), whose trajectory length (and therefore the reflection rule) is given by a norm with unit ball $T^{\circ}$ (polar to a body $T \subset V^{*}$ containing the origin); throughout this paper we use the possibly non-standard notation $\|\cdot\|_{T}$ for this norm with $T$ lying in the dual space.

We emphasize that in this work the norm need not be symmetric, that is, need not satisfy $\|q\|=\|-q\|$. Usually the term "Minkowski billiard" is used, but Minkowski norms are usually assumed to be symmetric, and we do not restrict ourselves to this particular case. The idea of [4] is to interpret a billiard trajectory in $K$ with norm $\|\cdot\|_{T}$ as a characteristic on the boundary of the convex body $K \times T \subset V \times V^{*}$. The space $V \times V^{*}$ is the cotangent bundle of $V$ and carries a natural symplectic structure, and the surface $\partial(K \times T)$, in a sense, carries a contact structure, although some effort has to be made to handle it because it is not smooth at $\partial K \times \partial T$.

Received by the editors April 5, 2015 and, in revised form, October 5, 2015 and December 14, 2015.

2010 Mathematics Subject Classification. Primary 52A20, 52A23, 53D35.

Key words and phrases. Billiards, Minkowski norm, Mahler's conjecture.

The first author was supported by the People Programme (Marie Curie Actions) of the European Union's Seventh Framework Programme (FP7/2007-2013) under REA grant agreement $\mathrm{n}^{\circ}[291734]$.

The first and third authors were supported by the Dynasty Foundation.

The first, second and third authors were supported by the Russian Foundation for Basic Research grant 15-31-20403 (mol_a_ved).

The second and third authors were supported by the Russian Foundation for Basic Research grant 15-01-99563 A. 
The symplectic approach was rather useful and gave certain results about the number $\xi_{T}(K)$, that is, the minimal $\|\cdot\|_{T}$-length of a closed billiard trajectory in $K$. In particular, in [4] this number was shown to be equal to the Hofer-Zehnder capacity $c_{H Z}(K \times T)$, and it was proved that the number $\xi_{T}(K)$ is monotone in $T$ and $K$ under inclusions and satisfies a certain Brunn-Minkowski type inequality. In [3] the inequality

$$
\xi_{K} \circ(K) \geq 4
$$

for centrally symmetric convex bodies was established with rather elementary techniques and it was noticed that, assuming the Viterbo conjecture for convex bodies $X \subset \mathbb{R}^{2 n}$,

$$
\operatorname{vol}(X) \geq \frac{c_{H Z}(X)^{n}}{n !},
$$

the estimate (1.1) would imply the famous Mahler conjecture [8]

$$
\operatorname{vol} K \cdot \operatorname{vol} K^{\circ} \geq \frac{4^{n}}{n !} \text {. }
$$

Mahler's conjecture is known so far in a weaker form with $\frac{\pi^{n}}{n !}$ on the right hand side; this is a result due to Greg Kuperberg [7]. More detailed information on this conjecture is given in the blog post [10] of Terence Tao and the paper [3]. For the Viterbo conjecture and its possible generalizations, we recommend the paper [1] and the references therein.

In this paper we invoke a more elementary and efficient approach, developed by Károly Bezdek and Daniel Bezdek in [5] for the Euclidean norm. It turns out that this approach remains valid without change for possibly asymmetric norms 1 ; it allows us to give elementary proofs of most results of [4, worry less about the non-smoothness issues, and establish the inequality

$$
\xi_{K^{\circ}}(K) \geq 2+2 / n
$$

for possibly non-symmetric convex bodies $K$ containing the origin. The latter inequality is related to the non-symmetric Mahler conjecture; see the discussion in Section 4 below.

\section{The BeZDEKS' APPROACH TO BILLIARDS}

Let us show how the results of 4 can be approached using the elementary technique of [5]. First, we consider an $n$-dimensional real vector space $V$, a convex body $K \subset V$, and define

$$
\begin{array}{r}
\mathcal{P}_{m}(K)=\left\{\left(q_{1}, \ldots, q_{m}\right):\left\{q_{1}, \ldots, q_{m}\right\} \text { does not fit into } \alpha K+t\right. \\
\text { with } \alpha \in(0,1), t \in V\} .
\end{array}
$$

Observe that "does not fit into $\alpha K+t$, with $\alpha \in(0,1), t \in V$ " is equivalent to "does not fit into the interior of $K+t$ with $t \in V$ ".

Then we consider a norm on $V$ such that the unit ball $T \subset V^{*}$ of its dual is smooth. We denote this norm by $\|\cdot\|_{T}$ following [4. Note that this norm need not be reversible in what follows, that is, $\|q\|_{T}$ need not be equal to $\|-q\|_{T}$.

\footnotetext{
${ }^{1}$ These ideas for the Euclidean norm in the plane first appeared in [6]; it was already mentioned there that more arbitrary distances (norms) can be considered similarly.
} 


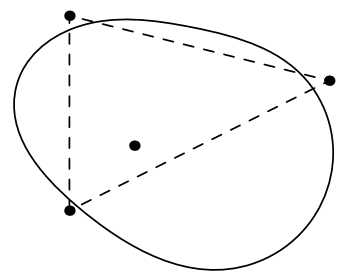

FiguRE 1. An element of $\mathcal{P}_{3}(K)$.

We define the length of the closed polygonal line as

$$
\ell_{T}\left(q_{1}, \ldots, q_{m}\right)=\sum_{i=1}^{m}\left\|q_{i+1}-q_{i}\right\|_{T},
$$

where indices are always modulo $m$. So the renovated result of [5] reads:

Theorem 2.1. For smooth convex bodies $K \subset V$ and $T \subset V^{*}$, the length of the shortest closed billiard trajectory in $K$ with norm $\|\cdot\|_{T}$ equals

$$
\xi_{T}(K)=\min _{m \geq 1} \min _{P \in \mathcal{P}_{m}(K)} \ell_{T}(P) .
$$

Moreover, the minimum is attained at $m \leq n+1$.

Remark 2.2. The right hand side of the above formula is well defined without any assumption on the smoothness of $K$ and $T$. In what follows we use it as the definition of $\xi_{T}(K)$ even when neither $K$ nor $T$ is smooth. It makes sense to call the minimizer in this theorem a shortest generalized billiard trajectory, which coincides with a shortest closed billiard trajectory in the case of smooth $K$ and $T$, as we will see from the proof of Theorem 2.1.

A shortest generalized billiard trajectory has the following geometrical meaning. Let $p$ be a non-smooth point of $\partial K$; we consider a trajectory $\ell$ through the point $p$ as a trajectory satisfying the reflection rule for some normal to $K$ at $p$, that is, we can take an arbitrary support hyperplane to $K$ at $p$ as if it were a tangent plane (Figure 2).

The shortest generalized billiard trajectory in an obtuse triangle is shown in Figure 3. It is a well-known open problem whether there is a legal (not passing through any vertex) closed billiard trajectory in every obtuse triangle.

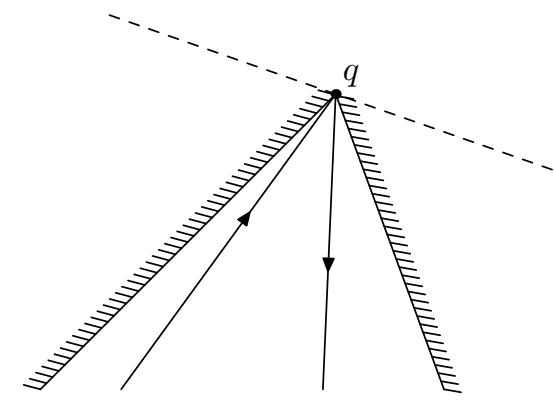

Figure 2. The reflection rule at a non-smooth point 


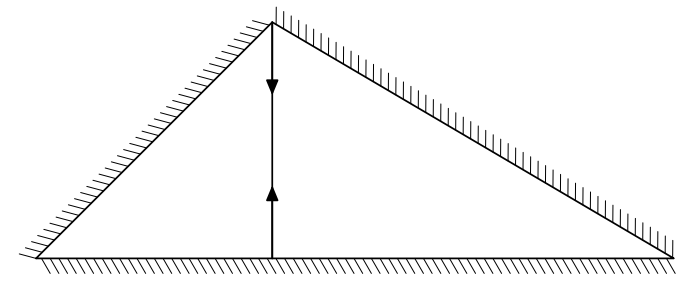

FiguRE 3. The shortest generalized billiard trajectory in an obtuse triangle

Proof of Theorem 2.1. The proof in [5, Lemma 2.4] is given for the Euclidean norm; the same argument works in this more general case. We reproduce the steps here.

First, let us recall the reflection rule (see [3], for example): For a billiard trajectory $\left\{q_{1}, \ldots, q_{m}\right\}$ we have in $V^{*}$ that

$$
p_{i+1}-p_{i}=-\lambda_{i} n_{K}\left(q_{i}\right), \quad \lambda_{i}>0 .
$$

This reflection rule is obtained by using the Lagrange multiplier method to optimize the expression $\left\|q_{i+1}-q_{i}\right\|_{T}+\left\|q_{i}-q_{i-1}\right\|_{T}$ varying $q_{i}$ under the assumption that $q_{i} \in \partial K$. There arise the momenta $p_{i}$ that are obtained from the velocities

$$
v_{i}=\frac{q_{i}-q_{i-1}}{\left\|q_{i}-q_{i-1}\right\|_{T}}
$$

by taking the differential $p=d\|v\|_{T}$ (recall that the differential is in the dual space). From this definition it follows that $p_{i} \in \partial T$, and if we want to go back and determine the velocity $v_{i}$, we just take

$$
v_{i}=d\left\|p_{i}\right\|_{T^{\circ}},
$$

resulting in $v_{i} \in \partial T^{\circ}$. Here we need the smoothness of $T$ to define velocities knowing momenta and the smoothness of $K$ to define the normals to $K$.

The normal $n_{K}$ at a boundary point of the convex body $K$ is also considered as a linear functional in $V^{*}$ of the unit norm, having maximum on $K$ precisely at this point. After summation over $i$ in (2.1) we obtain

$$
\sum_{i} \lambda_{i} n_{K}\left(q_{i}\right)=0
$$

that is, the normals at the bounce points $q_{i}$ surround the origin in $V^{*}$. This means that the set $\left\{q_{1}, \ldots, q_{m}\right\}$ cannot be covered by a smaller positive homothet of $K$. Indeed, assume that a homothet $\alpha K+t$ with $\alpha \in(0,1)$ covers all the points $\left\{q_{i}\right\}$, therefore the translate $K+t$ of $K$ contains $q_{i}$ 's in its interior; here we assume that the origin of $V$ is contained in $K$ without loss of generality. Let $n_{i}$ be the normal (linear form) such that

$$
\max _{q \in K}\left\langle n_{i}, q\right\rangle=\left\langle n_{i}, q_{i}\right\rangle .
$$

By the assumption that $\operatorname{int}(K+t) \ni q_{i}$,

$$
\left\langle n_{i}, t\right\rangle+\max _{q \in K}\left\langle n_{i}, q\right\rangle=\max _{q \in K}\left\langle n_{i}, q+t\right\rangle>\left\langle n_{i}, q_{i}\right\rangle=\max _{q \in K}\left\langle n_{i}, q\right\rangle,
$$

hence $\left\langle n_{i}, t\right\rangle>0$, and summing such inequalities, we obtain

$$
\left\langle\sum_{i} \lambda_{i} n_{i}, t\right\rangle=\langle 0, t\rangle>0,
$$


which is a contradiction. We conclude that a shortest closed billiard trajectory $Q_{\text {min }}=\left\{q_{1}^{\prime}, \ldots, q_{m^{\prime}}^{\prime}\right\}$ must be an element of some $\mathcal{P}_{m^{\prime}}(K)$.

Now we go in the opposite direction to consider a polygonal line $Q=\left\{q_{1}, \ldots, q_{m}\right\}$ $\in \mathcal{P}_{m}(K)$ on which the minimum is attained, including the minimum with respect to varying $m$. The previous paragraph shows that $\ell_{T}(Q) \leq \ell_{T}\left(Q_{\min }\right)$. Applying the Helly theorem, we readily see that we can replace $Q$ by a subset with at most $m \leq n+1$ points keeping the property of not fitting into a smaller homothet of $K$. In order to finish the proof, we must show that $Q$ is a generalized billiard trajectory on $K$.

We can find a translate $K+t$ that contains $Q$; such a translate must exist because otherwise we could take a smaller homothet of $Q$, still not fitting into the interior of $K$, so $Q$ would not be the length minimizer in $\mathcal{P}_{m}(K)$. By [5, Lemma 2.2], the assumption that $Q$ does not fit into a smaller homothet of $K$ is certified, possibly after omitting the $q_{i}$ lying in the interior of $K+t$, by considering a set of halfspaces $H_{i}^{+} \supseteq K+t$, with respective complementary halfspaces $H_{i}^{-}$supporting $K+t$ such that $q_{i} \in H_{i}^{-} \cap K$, and the intersection $\bigcap_{i=1}^{m} H_{i}^{+}$is nearly bounded (that is, lies between two parallel hyperplanes). This actually means that the outer normals $n_{i}$ to $K+t$ at the $q_{i}$ can be non-negatively combined to zero. From here on we assume without loss of generality that $t=0$ and write $K$ instead of $K+t$.

We then observe that varying the $q_{i}$ inside their respective $H_{i}^{-}$(and allowing them to get outside $K$ ) we never obtain a configuration that can be put into a smaller homothet of $K$, because a smaller homothet of $K$ has to miss some $H_{i}^{-}$. This is established by the same argument with normals surrounding the origin resulting in (2.2). Now let us try to minimize the length $\ell_{T}\left(q_{1}, \ldots, q_{m}\right)$ over

$$
\mathcal{H}=\left\{\left(q_{1}, \ldots, q_{m}\right): \forall i q_{i} \in H_{i}^{-}\right\} .
$$

We have shown that $\mathcal{H} \subseteq \mathcal{P}_{m}(K)$ and therefore $Q$ is also a length minimizer in $\mathcal{H}$. Now we conclude from minimizing the length that every $q_{i}$ must either be a "fake" vertex where $Q$ actually does not change its direction, or a vertex where $Q$ reflects from $H_{i}^{-}$according to (2.1); the latter is readily obtained with the Lagrange multiplier method from the minimal length assumption. The "fake" vertices may again be omitted keeping the property $Q \in \mathcal{P}_{m}(K)$ with $m \leq n+1$, since the triangle inequality holds for asymmetric norms as usual if we keep the order of the points. The reflection points $q_{i}$ are on $\partial K$, and the normals to $K$ at $q_{i}$ must equal the normals to the respective $H_{i}^{+}$. So we conclude that $Q$ is a billiard trajectory of $K$ obeying (2.1) and $\ell_{T}(Q) \geq \ell_{T}\left(Q_{\min }\right)$. Since the opposite inequality is established in the first half of the proof, the proof is complete.

\section{Derivation of Classical AND OF ONE NEW RESUlT}

3.1. Monotonicity of $\xi_{T}(K)$. Let us show how the results of [4] on the function $\xi_{T}(K)$ follow easily from Theorem 2.1. First, the monotonicity

$$
\xi_{T}(K) \leq \xi_{T}(L) \text { when } K \subseteq L
$$

follows easily because $\mathcal{P}_{m}(K) \supseteq \mathcal{P}_{m}(L)$ and the minimum can only get smaller on a larger set.

3.2. Symmetry. To prove the Brunn-Minkowski type inequality, as in [4, we need the following equality:

$$
\xi_{T}(K)=\xi_{K}(T)
$$


This is obvious in the symplectic approach; the idea 9 is essentially that closed billiard trajectories correspond to critical points of the action functional

$$
\sum_{i=1}^{m}\left\langle p_{i+1}, q_{i+1}-q_{i}\right\rangle=\sum_{i=1}^{m}\left\langle p_{i}-p_{i+1}, q_{i}\right\rangle
$$

with constraints $q_{1}, \ldots, q_{m} \in \partial K$ and $p_{1}, \ldots, p_{m} \in \partial T$, and the value of this functional at a critical point equals

$$
\sum_{i=1}^{m}\left\|q_{i+1}-q_{i}\right\|_{T}=\sum_{i=1}^{m}\left\|p_{i}-p_{i+1}\right\|_{K} .
$$

This argument uses the smoothness of $K$ and $T$ in an essential way, but the monotonicity property allows us to approximate any convex body by smooth bodies from below and from above, and then to pass to the limit.

3.3. Brunn-Minkowski-type inequality. Having noted all this, we observe that for the Minkowski sum $S+T$ in $V^{*}$ we have in $V$ :

$$
\|\cdot\|_{S+T}=\|\cdot\|_{S}+\|\cdot\|_{T} .
$$

Then it follows that

$$
\xi_{S+T}(K) \geq \xi_{S}(K)+\xi_{T}(K)
$$

because the minimum of the sum of functions is no less than the sum of the minima. After applying (3.2) this reads:

$$
\xi_{T}(K+L) \geq \xi_{T}(K)+\xi_{T}(L) .
$$

3.4. Estimates on $\xi_{T}(K)$. We can even prove something new with this technique, or the technique of $[$.

Definition 3.1. Following [5, we call $K$ 2-periodic with respect to $T$ if one of its shortest generalized billiard trajectories bounces on $\partial K$ only twice.

We recall the main result of [3]:

Theorem 3.2 (Artstein-Avidan, Karasev, Ostrover, 2013). If $K$ and $T$ are centrally symmetric and polar to each other $\left(T=K^{\circ}\right)$, then $\xi_{T}(K)=4 . K$ is 2 periodic with respect to $T$ and every segment $[-q, q]$, for any $q \in \partial K$, is a shortest generalized billiard trajectory.

Remark 3.3. There may be other minimal trajectories that are not 2-bouncing if $K$ is not strictly convex. This can be seen already for the square $K=[-1,1]^{2}$.

Having developed the appropriate technique, we give:

The short new proof of Theorem 3.2. Let us show that $\xi_{T}(K) \geq 4$. From Theorem 2.1 we conclude that it is sufficient to show that any closed polygonal line of length (in the given norm) less than 4 can be covered by an open unit ball. This is done with the well-known folklore argument that follows.

Assume a closed polygonal line $P$ has length less than 4 . Take points $x, y \in P$ that partition $P$ into two parts of equal lengths; each part will have length less than 2 (Figure 4). For any $z \in P$, lying in either of the parts, we compare the straight line segments and the segments of $P$ and deduce that

$$
\|z-x\|+\|z-y\|<2
$$

from the triangle inequality. 


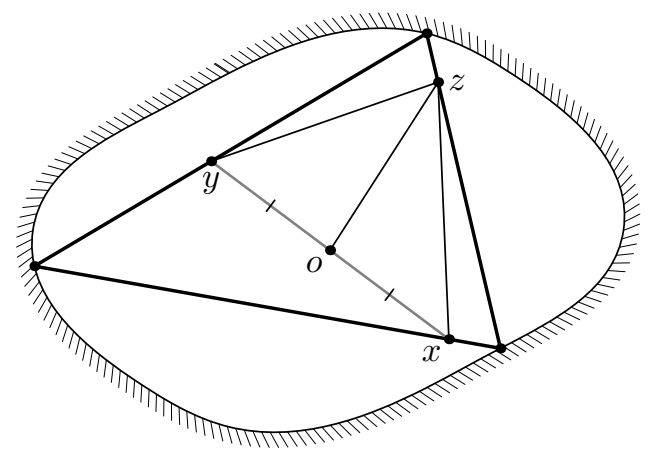

FiguRE 4. Explanation of the proof of Theorem 3.2

Let $o$ be the midpoint of the segment $[x y]$. From the triangle inequality we also have

$$
\|z-o\| \leq \frac{1}{2}(\|z-x\|+\|z-y\|)<1 .
$$

So we have proved that $P$ is covered by an open ball (a translate of the interior of $K$ ) with radius 1 centered at $o$. By Theorem 2.1, this is not a billiard trajectory in $K$.

So $\xi_{T}(K) \geq 4$ and actually the equality holds since every segment $[q,-q]$ with $q \in \partial K$ is a billiard trajectory of length 4 .

Remark 3.4. Let $K$ be strictly convex. If the length of $P$ were 4 , then in the above argument the equality $\|z-x\|+\|z-y\|=2$ will hold at most once on either half of $P$. So a translate of $K$ covers $K$ and $P$ has at most 2 bounces. Actually, one bounce is impossible, so a 2-bouncing trajectory is the only case of equality, and this trajectory must be the segment $[q,-q]$ for some $q \in \partial K$. If $K$ is not strictly convex, then other minimal trajectories also exist.

Remark 3.5. If $K$ is a square in the plane, which is not smooth and not strictly convex, then there are plenty of minimal trajectories. Here a minimal trajectory is understood as something providing the minimum to the right hand side of the defining equation in Theorem 2.1. Any segment connecting the two opposite sides of $K$ is such, and some of the quadrangles with vertices on the four sides of $K$ are also such.

As another simple exercise, we establish the following result:

Theorem 3.6. Let $K$ be 2-periodic with respect to $T$ and let $T$ be centrally symmetric. Then $K+\lambda T^{\circ}$ is also 2-periodic with respect to $T$ for any $\lambda$.

Proof. Consider one of the shortest closed billiard trajectories in $K$ bouncing at $q_{1}$ and $q_{2}$. From Theorem 3.2 we also know that $\xi_{T}\left(T^{\circ}\right)=4$ and we can find a pair $\{-q, q\} \in \partial T^{\circ}$ that gives a shortest closed billiard trajectory in $T^{\circ}$ with length 4 and such that $q$ is proportional to $q_{2}-q_{1}$. The minimality assumption for $\left\{q_{1}, q_{2}\right\}$ implies that the normals $-p$ and $p$ to $K$ at $q_{1}$ and $q_{2}$ are the same as the normals to $T^{\circ}$ at $-q$ and $q$ respectively.

Then the pair of points $\left\{q_{1}-\lambda q, q_{2}+\lambda q\right\}$ is in the boundary of $K+\lambda T^{\circ}$ and the normals to $K+\lambda T^{\circ}$ at these points are again $-p$ and $p$. Now it follows that 
$\left\{q_{1}-\lambda q, q_{2}+\lambda q\right\}$ is a closed billiard trajectory in $K+\lambda T^{\circ}$ of length $\xi_{T}(K)+\lambda \xi_{T}\left(T^{\circ}\right)$. From (3.3) it follows that this trajectory is minimal.

\section{Attempt toward the non-Symmetric Mahler's Conjecture}

In [3] Mahler's conjecture vol $K \cdot \operatorname{vol} K^{\circ} \geq \frac{4^{n}}{n !}$ for centrally symmetric convex $n$-dimensional $K$ was reduced, assuming the Viterbo conjecture on symplectic capacities, to proving that

$$
\xi_{K^{\circ}}(K) \geq 4
$$

which is true; see Theorem 3.2 in the previous section.

Dropping the assumption of the central symmetry, the corresponding version of Mahler's conjecture becomes (see [2])

$$
\operatorname{vol} K \cdot \operatorname{vol} K^{\circ} \geq \frac{(n+1)^{n+1}}{(n !)^{2}}
$$

for convex bodies $K \subset \mathbb{R}^{n}$ containing the origin in the interior. Again, assuming Viterbo's conjecture, in order to deduce from it the non-symmetric Mahler conjecture, one would have to prove that

$$
\xi_{K^{\circ}}(K) \geq\left(\frac{(n+1)^{n+1}}{n !}\right)^{1 / n},
$$

the right hand side being asymptotically $e$ by Stirling's formula. In fact, already for $n=2$ it is easy to check by hand, or look at Theorem 4.1 below, that the sharp estimate is

while (4.1) gives the number

$$
\xi_{K} \circ(K) \geq 3
$$

$$
\left(\frac{3^{3}}{2}\right)^{1 / 2}
$$

which is greater than 3. For higher dimensions, there also remains a gap between the actual lower bound for the billiard trajectory length and the bound needed to establish the non-symmetric Mahler conjecture, assuming the Viterbo conjecture.

Namely, we are going to prove:

Theorem 4.1. If $K \subset \mathbb{R}^{n}$ is a convex body containing the origin in its interior, then

and the bound is sharp.

$$
\xi_{K^{\circ}}(K) \geq 2+2 / n
$$

This theorem shows that the non-symmetric Mahler conjecture is out of reach of the billiard approach of [3].

Proof. We invoke Theorem 2.1 and consider a closed polygonal line $P$ not fitting into a smaller homothet of $K$. By the same theorem we can assume that $P$ has vertices $q_{1}, \ldots, q_{m}$ with $m \leq n+1$.

Observe that the norm $\|w\|_{K^{\circ}}$ of a vector $w \in V$ has a very simple meaning: Let $v \in \partial K$ be the vector positively proportional to $w$ and take

$$
\|w\|_{K^{\circ}}=\frac{|w|}{|v|}
$$

using the standard Euclidean norm $|\cdot|$ (Figure 5). Now to measure the length of $P$ we take $v_{1}, \ldots, v_{m} \in \partial K$ to be positively proportional to $q_{2}-q_{1}, \ldots, q_{1}-q_{m}$ 


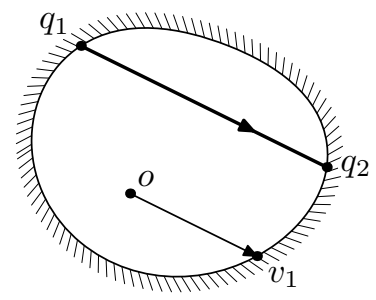

Figure 5. Measuring the length of a directed segment
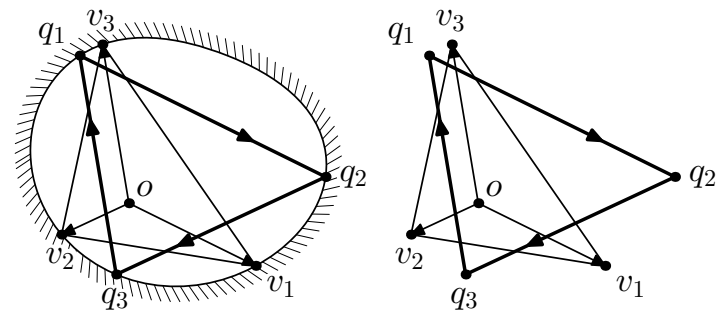

Figure 6. Replacing $K$ with $L$

respectively; it follows that the origin can be expressed as a positive combination of the vectors $\left\{v_{i}\right\}_{i=1}^{m}$. If we replace $K$ by the body $L=\operatorname{conv}\left\{v_{i}\right\}_{i=1}^{m}$ of possibly smaller dimension, then it is easy to see that $L$ still contains the origin and

$$
\ell_{K^{\circ}}(P)=\ell_{L^{\circ}}(P)
$$

since the $v_{i}$ 's are still on the boundary of $L$ (Figure 6). Moreover, $P$ cannot fit into a smaller homothet of $L$, since it does not fit into a smaller homothet of the larger body $K \supseteq L$. In this $\operatorname{argument} \operatorname{dim} L$ may become less than $\operatorname{dim} K$; in this case we use induction on dimension, since we have the monotonicity of the estimate $2+2 /(n-1)>2+2 / n$. The other case $\operatorname{dim} K=\operatorname{dim} L=n$ is only possible when $m=n+1$. We can therefore assume from the start that $L$ is a simplex.

Now we are in the following situation, changing the indexing of vertices slightly. $L$ is a simplex with vertices $v_{0}, \ldots, v_{n}$ and their respective opposite facets $F_{0}, \ldots, F_{n}$, and $P$ is a closed polygonal line with vertices $q_{0}, \ldots, q_{n}$. From the first step of our construction, the following relations hold:

$$
q_{i+1}-q_{i}=t_{i} v_{i}, \quad t_{i}>0 .
$$

Also, we can assume that $q_{0}, \ldots, q_{n}$ lie on the boundary of $L$, otherwise we can translate $P$ and inflate $L$, keeping the condition that $P$ does not fit into a smaller homothet of $L$, having eventually $P \subseteq L$ (Figure 7). By this the quantity $\ell_{L^{\circ}}(P)$ may only become smaller, and either all the vertices of $P$ will be on $\partial L$ or the dimension will drop and we use induction.

So either we use induction and drop the dimension of $L$, or we use (4.2) to conclude that the segment $\left[q_{i}, q_{i+1}\right]$ has direction $v_{i}$, the vector from the origin to a vertex of $L$. The latter implies that, if we look at $L$ along the line of sight $v_{i}$, then we see the facet $F_{i}$ and (strictly) do not see the other facets. Therefore the segment $\left[q_{i}, q_{i+1}\right]$ must start at $F_{i}$ and point into the interior of $L$, its endpoint $q_{i+1}$ must 


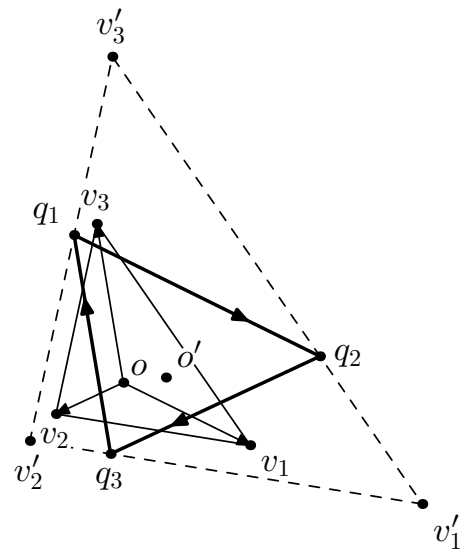

Figure 7 . The inflation of $L$

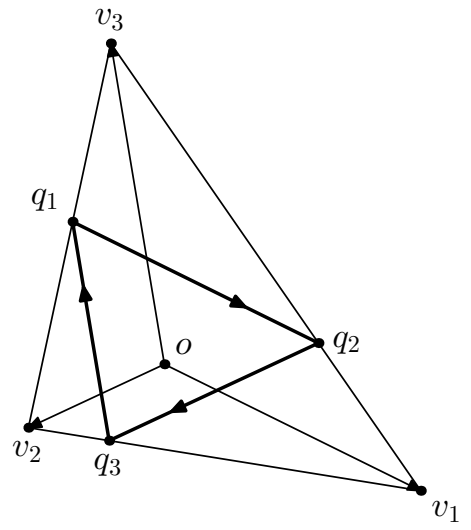

Figure 8. A billiard trajectory in the triangle

lie on some other $F_{j}(j \neq i)$, and if we extend this segment to a half-line beyond $q_{i+1}$, it must leave $L$ at $q_{i+1}$. Assuming $q_{i} \neq q_{i+1}$ (otherwise we have less points and the dimension drops) we obtain, in particular, that the point $q_{i}$ can only lie in the relative interior of its respective $F_{i}$.

We see that $q_{i}$ is the projection of $q_{i+1}$ onto $F_{i}$ parallel to $v_{i}$. If we apply these projections cyclically starting from $q_{i} \in F_{i}$ and ending at the same point, then we obtain a map that takes $F_{i}$ into its relative interior and that is linear on $F_{i}$. Such a map has a unique fixed point. So it follows that having chosen $L$ with a cyclic order on its facets we can reconstruct the considered polygonal line $P$ uniquely.

Another way to show the uniqueness is to observe that the condition (4.2) implies $\sum_{i=0}^{n} t_{i} v_{i}=0$ and therefore determines the $t_{i}$ uniquely up to a positive multiple. Hence the polygonal line $P$ is determined uniquely up to translation and a positive homothety, and the additional property $q_{i} \in F_{i}$ fixes it completely.

Now we are going to consider everything in barycentric coordinates. Let $\left(m_{0}, \ldots, m_{n}\right)$ be the barycentric coordinate of the origin in $L$. Then it is not hard to express the $q_{i}$ in terms of the $v_{i}$. We are going to index everything cyclically modulo $n+1$ and we put

$$
M=\sum_{0 \leq k<l \leq n} m_{k} m_{l} .
$$

From the Schur concavity of the elementary symmetric functions, it follows that $M$ takes its maximum value at $m_{0}=\cdots=m_{n}=\frac{1}{n+1}$ and therefore $M \leq \frac{n}{2 n+2}$. We have already shown the uniqueness of the $q_{i}$ after the choice of the order of the projections along the $v_{i}$ to facets. It remains to guess the expression for $q_{i}$ and prove that it gives the solution. Our guess is

$$
q_{i}=\frac{\sum_{j \neq i} \sum_{k=i}^{j-1} m_{j} m_{k} v_{j}}{M},
$$

where the inner summation goes cyclically from $i$ to $j-1$, so it is allowed that $j-1<i$. First, it is easy to observe that the sum of all coefficients in the numerator equals $M$, because every monomial $m_{k} m_{l}$ is used precisely once. Therefore we have 


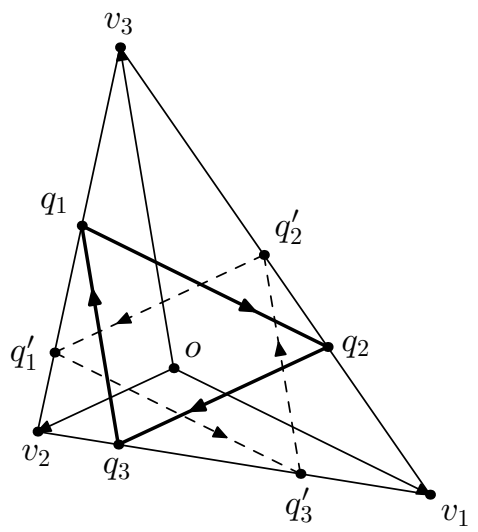

Figure 9. The two trajectories in the two-dimensional triangle

$q_{i} \in F_{i}$. Then we express the vector $q_{i+1}-q_{i}$ as

$$
q_{i+1}-q_{i}=\frac{\sum_{j \neq i+1} \sum_{k=i+1}^{j-1} m_{j} m_{k} v_{j}-\sum_{j \neq i} \sum_{k=i}^{j-1} m_{j} m_{k} v_{j}}{M}=\frac{\sum_{j \neq i} m_{i} m_{j} v_{i}-\sum_{j \neq i} m_{j} m_{i} v_{j}}{M} .
$$

Since $\sum m_{j} v_{j}=0$, we obtain $\sum_{j \neq i} m_{j} m_{i} v_{j}=-m_{i}^{2} v_{i}$. And from $\sum_{j} m_{j}=1$ we get $\sum_{j \neq i} m_{i} m_{j} v_{i}+m_{i}^{2} v_{i}=m_{i} v_{i}$. Finally,

$$
q_{i+1}-q_{i}=\frac{m_{i} v_{i}}{M} \text { and } t_{i}=\frac{m_{i}}{M} .
$$

Now we can bound the sum of $t_{i}$ from below,

$$
\sum_{i} t_{i}=\sum_{i} \frac{m_{i}}{M}=\frac{1}{M} \geq \frac{2 n+2}{n} .
$$

This means that the length of $P$ in the norm with unit ball $L$ is at least $2+2 / n$, and with all $m_{i}$ equal this bound is actually attained.

Since it is possible to approximate $L$ by a smooth body, whose polar is also smooth, keeping the trajectory and its length the same, we conclude that the bound is sharp even in the class of smooth bodies $K$ with smooth polars.

Remark 4.2. A more rigorous analysis of the trajectory $q_{1} \ldots q_{n+1}$ (Figure 8) shows that a trajectory in the simplex passing through every facet is locally minimal if and only if its segments are parallel to the segments $o v_{i}$ in some order.

One curious thing follows from the proof of the theorem. If we fix a simplex $L$ with the origin inside, then there are $(n-1)$ ! cyclic orders on the vertices, and therefore $(n-1)$ ! trajectories inscribed in it with edges parallel to the respective vectors $v_{i}$ (Figure 9 ). These (billiard) trajectories are evidently different, but all corresponding edges in all the trajectories have the same length. 
One consequence of this observation is that if we consider a trajectory $q_{0} \ldots q_{n}$ and draw the hyperplane $h_{i}$ through the midpoint of every segment $q_{i} q_{i+1}$, parallel to the facet $F_{i}$ of $L$, then all these hyperplanes $h_{i}$ intersect in a single point.

The proof of Theorem 4.1 also reveals the following formula: Let $\ell_{i}$ be the length of the Cevian 2 of $L$ passing through the vertex $v_{i}$ and the origin. Then for any closed polygonal line $P=\left(q_{0}, \ldots, q_{n}\right)$ with $q_{i} \in F_{i}$ and $q_{i+1}-q_{i}=t_{i} v_{i}$ with $t_{i}>0$, we have

$$
\sum \frac{\left|q_{i+1}-q_{i}\right|}{\ell_{i}}=2
$$

Indeed, $\frac{\left|v_{i}\right|}{\ell_{i}}=\sum_{j \neq i} m_{j}$, since the $m_{i}$ are the barycentric coordinates of the origin. So we obtain

$$
\sum \frac{\left|q_{i+1}-q_{i}\right|}{\ell_{i}}=\frac{\sum_{i} m_{i}\left(\sum_{j \neq i} m_{j}\right)}{M}=\frac{2 M}{M}=2 .
$$

\section{ACKNOWLEDGMENTS.}

The authors thank Yaron Ostrover for numerous remarks and corrections and the anonymous referee for many corrections which helped us improve the text.

\section{REFERENCES}

[1] J. C. Álvarez Paiva and F. Balacheff, Contact geometry and isosystolic inequalities, Geom. Funct. Anal. 24 (2014), no. 2, 648-669, DOI 10.1007/s00039-014-0250-2. MR3192037

[2] J.-C. Álvarez Paiva, F. Balacheff, and K. Tzanev, Isosystolic inequalities for optical hypersurfaces, 2013, arXiv:1308.5522

[3] Shiri Artstein-Avidan, Roman Karasev, and Yaron Ostrover, From symplectic measurements to the Mahler conjecture, Duke Math. J. 163 (2014), no. 11, 2003-2022, DOI 10.1215/00127094-2794999. MR.3263026

[4] Shiri Artstein-Avidan and Yaron Ostrover, Bounds for Minkowski billiard trajectories in convex bodies, Int. Math. Res. Not. IMRN 1 (2014), 165-193. MR3158530

[5] Dániel Bezdek and Károly Bezdek, Shortest billiard trajectories, Geom. Dedicata 141 (2009), 197-206, DOI 10.1007/s10711-009-9353-6. MR2520072(2010h:52009)

[6] K. Bezdek and R. Connelly, Covering curves by translates of a convex set, Amer. Math. Monthly 96 (1989), no. 9, 789-806, DOI 10.2307/2324841. MR1033346 (90k:52020)

[7] Greg Kuperberg, From the Mahler conjecture to Gauss linking integrals, Geom. Funct. Anal. 18 (2008), no. 3, 870-892, DOI 10.1007/s00039-008-0669-4. MR2438998(2009i:52005)

[8] Kurt Mahler, Ein Übertragungsprinzip für konvexe Körper (German), Casopis Pěst. Mat. Fys. 68 (1939), 93-102. MR0001242 (1,202c)

[9] Serge Tabachnikov, Geometry and billiards, Student Mathematical Library, vol. 30, American Mathematical Society, Providence, RI; Mathematics Advanced Study Semesters, University Park, PA, 2005. MR2168892 (2006h:51001)

\footnotetext{
${ }^{2}$ Cevians of a simplex $L$ are $n+1$ segments connecting the vertices $v_{i}$ with their respective opposite facets $F_{i}$ and all having a common point in the interior of $L$.
} 
[10] T. Tao, Open question: The Mahler conjecture on convex bodies, 2007, terrytao.wordpress.com

Institute of Science and Technology Austria (IST Austria), Am Campus 1, 3400 Klosterneuburg, Austria - And - Institute for Information Transmission Problems Ras, Bolshoy Karetny Per. 19, Moscow, Russia 127994

E-mail address: akopjan@gmail.com

Moscow Institute of Physics and Technology, Institutskiy per. 9, Dolgoprudny, Russia 141700 - AND - Institute for Information Transmission Problems RAS, Bolshoy KAREtny PER. 19, Moscow, Russia 127994

E-mail address: alexey_m39@mail.ru

Moscow Institute of Physics And Technology, Institutskiy per. 9, Dolgoprudny, Russia 141700 - And - Institute for Information Transmission Problems RAS, Bolshoy KARETny PER. 19, Moscow, Russia 127994

E-mail address: r_n_karasev@mail.ru

URL: http://www.rkarasev.ru/en/

Moscow Institute of Physics And Technology, Institutskiy Per. 9, Dolgoprudny, Russia 141700

E-mail address: independsharik@yandex.ru 